\title{
Éducation à la diversité et langues immigrées : expériences européennes
}

Andrée Tabouret-Keller

\section{(2) OpenEdition \\ 12 Journals}

Édition électronique

URL : http://journals.openedition.org/esp/969

DOI : 10.4000/esp.969

ISSN : 2532-0319

Éditeur

Centre d'Information sur l'Éducation Bilingue et Plurilingue

\section{Édition imprimée}

Date de publication : 1 décembre 2016

Pagination : 89-102

ISSN : 1127-266X

\section{Référence électronique}

Andrée Tabouret-Keller, «Éducation à la diversité et langues immigrées : expériences européennes », Éducation et sociétés plurilingues [En ligne], 41 | 2016, mis en ligne le 20 octobre 2017, consulté le 24 septembre 2020. URL : http://journals.openedition.org/esp/969 ; DOI : https://doi.org/10.4000/esp. 969 


\section{Éducation à la diversité et langues immigrées: expériences européennes. Philippe BLANCHET et Stéphanie GLERG CONAN (co-ord.). Migrations Société, Dossier (p. 48-183), vol. 27, $\mathrm{n}^{\circ} 162,2015$.}

\section{Andrée Tabouret-Keller}

Gli autori presentano $i$ testi riuniti in questo dossier come esperienze concrete elaborate e valutate grazie ad un lavoro rigoroso il cui scopo è quello di condividere interrogativi pedagogici al fine di contribuire ad un interrogativo globale sui rapporti fra immigrazione e società in Europa.

Parole chiave: rivista Migrations-Société; lavoro di terreno; pedagogia; immigazione; diversità linguistica

The authors present the texts of this Dossier as "concrete experiences, conceived and evaluated systematically and rigorously", whose aim it is "to share pedagogical issues and contribute to a global questioning of relations between immigration and society" in Europe.

Key-words: journal Migrations-Société, fieldwork, pedagogy, immigration, language diversity

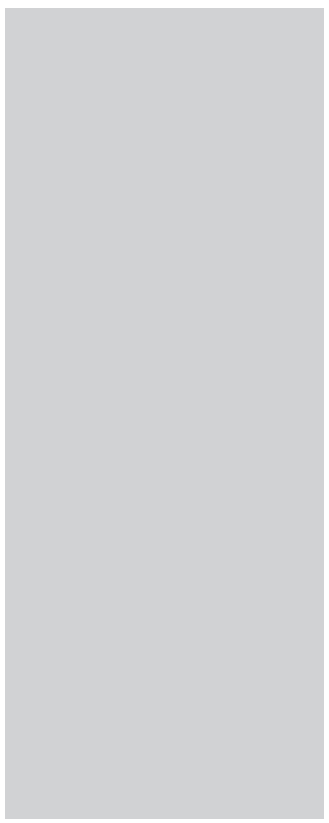

«Passer de l'exclusion à l’inclusion: des expériences réussies d'éducation à et par la diversité linguistique à l'école», Philippe BLANCHET et Stéphanie CLERC CONAN (p. 51-70).

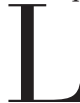

a prise en compte des questions sociolinguistiques liées aux phénomènes d'immigration en Europe s'est appuyée sur le renforcement de la centration sur la lange unique dite nationale ou officielle de la part des États et d'une part majoritaire des opinions publiques en même temps qu'elle recommandait la production d'outils didactiques pour le maintien et le développement du plurilinguisme dans ces États et notamment des populations immigrées, de la part des chercheurs, des éducateurs, d'organismes à vocation éducative et culturelle comme le Conseil d' l'Europe. A partir des années 1950 et surtout 1970, des voix se sont élevées pour faire valoir les droits linguistiques comme des droits humains fondamentaux et leur violation comme des discriminations. Cette prise de conscience a suscité le développement des études interculturelles en montrant l'impossibilité effective de l'assimilation (y compris pour les «régionaux») et en proposant de penser une intégration de type interculturel en associant les cultures d'origine (dont les langues) et la ou les cultures des pays d'accueil, via des formes de métissage (y compris linguistique). Une telle transformation doit être accompagnée par une éducation qu'on appellera interculturelle, puis également plurilingue, donc par un rapport diffé- 
Compte-rendu

A. TABOURET-Keller rent et positif à la diversité linguistique et culturelle aux niveaux collectif et individuel: on passe de la connaissance de la langue à la compétence à communiquer, le Cadre européen commun de référence pour les langues (CECRL, Conseil de l'Europe, 1997), le Cadre de référence pour les approches plurielles (CARAP, Conseil de l'Europe, 2001) y insistent. Ce développement est freiné par le renforcement des idéologies nationales, voire nationalistes, par la «droitisation» des sociétés qui s'accentue sur les plans de l'idéologie économique dite «néolibérale» et de l'idéologie identitaire. A quoi s'ajoute l'absence d'outils, de formation, de connaissances concernant la mise en place d'une éducation plurilingue inclusive. Le présent dossier illustre les fondements scientifiques, les modalités pratiques et les effets dans divers contextes d'une telle éducation en présentant des expériences incluant la formation des enseignants, des pratiques de classe, des outils précis, des résultats observés, les moyens d'élaboration, de suivi et d'évaluation des dispositifs mis en œuvre dans différents pays européens (Allemagne, Belgique, France, Grande Bretagne, Grèce et Portugal).

Les auteurs exemplifient leur analyse à partir de remarques sur la situation en France, par un bref rappel historique, de l'ELCO à la fin des années 1970 (enseignement des langues et cultures d'origine) au FLI des années 2010 (français langue d'intégration) et aux nouveaux programmes proposés en 2015 pour le cycle 4 dont le socle commun concerne trois domaines: «les langages pour penser et communiquer», «les méthodes et outils pour appendre», «la formation de la personne». Ils présentent les textes réunis dans ce Dossier comme «des expériences concrètes élaborées et évaluées par un travail rigoureux» (p. 65) dont le propos est de «partager des questionnements pédagogiques pour contribuer à un questionnement global sur les rapports entre immigration et société» et la présentation rapide des six contributions au Dossier, que je présente séparément ici. Ces dispositifs éducatifs ne sont pas présentés en insistant sur les questions techniques d'«enseignementapprentissage» des langues et des cultures mais sur des projets de transformation des modalités et finalités éducatives en contexte plurilingue et interculturel de sorte à transformer le rapport entretenu avec l'immigration dans les sociétés. «C'est la raison pour laquelle le présent Dossier a semblé plus à sa place dans une revue interdisciplinaire à large empan sur les questions sociétales liées aux immigrations que dans une revue disciplinaire de didactique des langues» (p. 66).

«Les acteurs éducatifs scolaires dans un projet de recherche doctorale d'éducation à la diversité à travers les langues-cultures en cycle 2», Éric BRUN (p. 71-84).

Cette contribution fait le point sur quatre années de recherche doctorale dont l'objet a été l'implantation et le développement triennal du pro- 
Compte-rendu

A. TABOURET-Keller jet EALCM, «éveil aux langues et aux cultures du monde» en cycle 2 dans deux écoles du Var, entre 2011 et 2015. Les principaux acteurs éducatifs concernés sont les familles, les enseignantes (toutes des femmes), l'agent territorial, les conseillères pédagogiques, les inspectrices, les animateurs; ce sont eux qui décident, organisent, gèrent et construisent les apprentissages scolaires des enfants. La méthodologie de la recherche est orientée par l'observation des dispositions attitudinales des enfants et de leurs enseignantes à l'égard de la différence/diversité à travers leur expression orale dans l'espace de la classe au cours de leurs interactions verbales. Les enfants ne sont pas représentés individuellement en raison de leur surreprésentation mais de leur enthousiasme pour chaque activité de découverte d'une autre langue-culture que la leur. Les acteurs éducatifs sont présentés dans l'ordre où ils figurent ci-dessus.

Les familles dans les interactions avec l'école: l'esprit du processus éducatif. Si le lien entre famille et école est relativement entretenu en maternelle, il est presque inexistant dès la première année de l'école primaire: le rôle qui incombe généralement aux familles se limite à un déplacement sécuritaire puisqu'elles ne sont pas - le plus souvent - conviées à pénétrer dans la classe pour y rester un moment auprès de l'enfant, un portail marquant physiquement la frontière du CP avec l'extérieur. La dichotomie opérée alors entre la famille et l'école, c'est-à-dire entre les parents et les enseignants, modélise un statut illusoire de l'enfant. L'enfant a changé de statut pour devenir un écolier parmi d'autres, il perçoit très vite ce rapport de forces qui est en jeu par la soumission de ses parents à l'autorité de l'école. Le projet EALCM vise une éducation mais vise aussi et surtout l'inclusion des familles en tant que véritables partenaires du processus éducatif.

Les enseignantes: le cour du processus éducatif. La difficulté d’implanter le projet EALCM repose sur la diversité même des enseignantes qui compose au sein d'une même école la longue chaine éducative du premier degré où chacune se positionne en fonction de ses expériences personnelles. Le taux d'adhésion ou de résistance au projet EALCM n'est généralement pas perçu comme une riche mosaique mais plutôt comme un patchwork plus ou moins bien assemblé.

Le premier dépend de la municipalité, le second de l'Éducation nationale. Les deux peuvent s'insérer dans le processus éducatif soit en tant que simples observateurs soumis à l'autorité du maître, soit en tant que participants actifs ayant certaines prérogatives sur l'éducation du groupe. D'après les observations faites au cours de cette recherche, l'ATSEM plus que l'AVS assume un rôle exclusivement disciplinaire pour soutenir tacitement l'enseignant dans ses activités et l'autorité de l'institution. 
Compte-rendu

A. TABouret-Keller

L'AGENT
TERRITORIAL
SPÉCIALISÉ
DE L'ÉCOLE
MATERNELLE
(ATSEM) ET
L'AUXILIAIRE DE VIE
SCOLAIRE (AVS):
LES MEMBRES
DU PROCESSUS
ÉDUCATIF.

Les conseillers pédagogiques d'éducation et les conseillers pédagogiques de langues vivantes: la coordination du processus éducatif. La présence d'un conseiller pédagogique de langues vivantes dès la maternelle serait fondamentale pour préparer les équipes pédagogiques en synergie avec celles de l'école élémentaire pour assurer un continuum cohérent en didactique de langue-culture. Mais le processus de remonter de l'information de terrain, c'est-à-dire la transmission de données, demeure défaillant.

Les inspecteurs de l'Éducation nationale de circonscription: l'encéphale du processus éducatif. Ils conçoivent leur fonction comme une gestion de micro-entreprise constituée par chacune des écoles. Il a été observé que les équipes pédagogiques assument le contrôle de l'école dans une logique autarcique, en rompant tout lien avec l'extérieur.

Les animateurs des activités périscolaires: les poumons du processus éducatif. Ils sont les seuls à traverser quasi librement l'espace scolaire en conservant leur multi-connexions avec l'extérieur (associations, clubs, etc.). Au cours de cette recherche leur statut a radicalement évolué: ces différents acteurs éducatifs ont aujourd'hui la possibilité d'occuper l'espace scolaire par intermittence sans lui appartenir totalement, sans obligation de lien avec les équipes pédagogiques ni de formation universitaire spécifique. Conclusion. Une des pistes conclusives qui n'était pas prévue est l'action prépondérante que jouent tous les acteurs scolaires adultes et chacun d'eux impliqués dans le processus éducatif. De nombreux obstacles entravent encore la réalisation du projet EALCM: il ne s'agit pas d'une réévaluation du sens moderne de l'éducation mais de la recherche du bien-être biologique de l'enfant. Les balbutiements d'une éducation à la diversité encore présents permettent de déconstruire petit à petit des systèmes représentationnels individuels et collectifs ethnocentristes pour reconstruire des liens plus altéritaires.

«Former aux concepts et à la pratique de l'éveil aux langues pour l'animation périscolaire: l'expérience de l'association DULALA», Elsa BEZAULT, Michel CANDELIER (p. 85-99).

L'expérience de l'association D'Une Langue À L'Autre, créée en 2009, œuvre pour la valorisation de toutes les formes de bilinguisme et pour le développement d'une éducation au plurilinguisme pour tous, elle développe principalement ses actions en Île-de-France (quatre salariés permanents à Montreuil) mais également en région lyonnaise. En 2014, plus de 1400 enfants et près de 4000 adultes ont été concernés par ses actions.

Les ateliers périscolaires. Leur objectif est de permettre à tous les enfants d'accéder «à des activités sportives, culturelles, artistiques». DULALA s'inspire du programme EVLANG (Union européenne, 1997-2001) qui valorise l'approche ludique ainsi que l'interdisciplinarité (expression corporelle, thêâtre, arts plastiques). 
Compte-rendu

A. TABouret-Keller
Les animatrices sont toutes des professionnelles de l'animation qui sont également enseignantes de leur propre langue maternelle, comédiennes, conteuses, auteures, artistes plasticiennes, elles adhèrent aux valeurs propres des ateliers: respect de chacun, participation de tous, utilisation de méthodes non-formelles, conviction profonde que tous les temps éducatifs doivent permettre aux enfants de développer des savoirs, des savoir-être et des savoir-faire. Pour les animatrices, habituées à travailler uniquement à partir de leur langue première et en français, l'éveil aux langues qui implique la présence et le mise en relation d'une grande diversité de langues, constitue un nouveau défi.

La formation est découpée en trois modules dont pendant trois mois les deux premiers sont centrés sur la présentation générale de l'éveil aux langues, les représentations des animatrices concernant les langues et cultures, la notion de compréhension plurilingue ainsi que sur un questionnement sur les modes de transmission et la posture de l'animateur: gestion de groupe et analyse, création d'activités d'éveil aux langues. Deux mois après, le troisième module est consacré à des approfondissements portant sur le rôle de l'animateur, à la méthode inductive, à la construction des séances.

La recherche pour élaborer une hypothèse concernant la chronologie des étapes générales de la formation a rencontré des difficultés d'organisation.

Le guide d'entretien s'articule en quatre étapes: 1. la connaissance préalable de l'animatrice sur la méthode d'éveil aux langues, 2. les représentations des animatrices sur ce qu'est l'éveil aux langues dans le cadre des ateliers DULALA, 3. les caractéristiques mentionnées de la pratique, 4. les expériences antérieures et l'évolution de la pratique. Ce guide est suivi de la présentation de trois cas: 1. l'évolution de MarieRose, 2. l'évolution de Zehra, 3. l'évolution de Nadia, qui illustrent les quatre étapes mentionnées par le guide.

Le bilan, sans vouloir tirer de conclusion définitive, souligne que l'éveil aux langues est en lien direct avec le «connu» dont disposent les animatrices, dont l'histoire personnelle s’impose comme le facteur déterminant de l'appropriation visée.

«Le rôle des langues d'origine dans la compétence plurilingue des jeunes issus de l'immigration: une étude de cas dans les écoles au Portugal», Rosa Maria FANECA, Maria Helena ARAÚJO e SÁ, Silvia MELO-PFEIFER (p. 101-120).

Au cours de la dernière décennie, le Portugal a accueilli des immigrés provenant de 179 pays des différents continents rejoints par leurs familles dont les enfants se sont inscrits à l'école. Leur niveau de maitrise des langues d'origine ainsi que du portugais témoigne d'une grande hétérogénéité. Les fonctions du portugais ont évolué: devenu langue 
Compte-rendu

A. TABOURET-Keller d'accueil il assume une fonction d'intégration pour de nombreux élèves. Malgré le développement de politiques linguistiques et éducatives promu par le Ministère (2005) les enseignants se heurtent à la complexité des situations marquée par la pluralité des langues, des cultures et des identités des apprenants pour lesquels le chemin de la réussite scolaire est à construire de manière singulière et inventive.

La première partie de l'étude concerne la «Contextualisation théorique» et présente les concepts qui sont au centre de la recherche:

Les langues d'origine: des concepts tels que «langue d'origine», «langue maternelle», «lange seconde», «langue d'accueil» sont souvent utilisés de manière floue et indissociable, ils sont évolutifs et instables bien qu'ils aient un impact sur la (dé)valorisation pédagogique des langues pratiquées par les enfants d'immigrés. Le concept de langue d'origine est chargé d'ambiguité car il renvoie aux racines, au contexte social, au pays d'origine des parents et à l'histoire de l'immigration, il est associé à une langue minoritaire en raison du nombre réduit de locuteurs dans le pays d'accueil, sa validité dépend d'un contexte sociologiques donné, régi par des paramètres tels que le manque ou la présence de politiques éducatives en faveur du plurilinguisme;

La compétence plurilingue et pluiculturelle: elle peut s'articuler autour des quatre dimensions suivantes: affective, linguistique et communicative, cognitive-verbale et une dimension liée à la gestion de l'interaction dans une perspective éminemment individuelle et cognitiviste, l'accent étant mis sur la mobilisation et l'interaction entre les connaissances linguistiques et communicationnelles déjà existantes des élèves, sur la gestion de leur répertoire affectif, cognitif et d'apprentissage en situation de communication.

La seconde partie est consacrée à l'étude empirique: méthodologie et recueil de données; cette étude s’insère dans une recherche plus vaste «Le rôle des langues d'origine dans la compétence plurilingue des jeunes issus de l'ímmigration: une étude de cas dans les écoles du district d'Aveiro et de Coimbra» (janvier 2012-décembre 2015). La première phase proposait un questionnaire à 139 élèves. La deuxième phase décrite ici consiste en des entretiens biographiques auprès d'élèves de 13 à 18 ans sélectionnés en tenant compte du contexte de la migration et de l'autorisation des parents pour participer aux entretiens qui doivent rendre compte de la conscience qu'a l'élève de la constitution de son répertoire plurilingue et des pratiques sociales qui lui sont associées et contribuent à son développement. Chaque entretien comporte six sections «Objectifs et questions des entretiens biographiques» dont trois sont détaillées dans le Tableau 1. Le Tableau 2 présente les «caractéristiques» de 20 des sujets interrogés maitrisant une, deux, voire trois langues d'origine ainsi que le portugais et deux ou trois langues étran- 
Compte-rendu

A. TABOURET-Keller gères.

Dans la Présentation et discussion des résultats, un certain nombre d'extraits d'entretiens suffisamment riches et très vivants servent à exemplifier et à complexifier la problématique de la recherche selon trois types de répertoires: socio-affectifs, linguistico-communicationnel et cognitifsverbaux et selon une analyse de la gestion de l'interaction

Les Considérations finales rappellent que le contexte scolaire place les langues d'origine dans un statut complexe et fluctuant entre langue maternelle, langue seconde et langue étrangère. La dimension émotionnelle et affective joue pour la majorité de ces jeunes un rôle majeur dans leurs relations aux différentes langues qu'ils ont à utiliser; leurs répertoires linguistiques reflètent leurs parcours pluriels et fragmentés ainsi que les contextes de leurs pratiques sociales où ils évoluent et où les langues apparaissent, disparaissent, se mélangent et/ou sont incorporées dans leur répertoire plurilingue. Il convient de noter que les entretiens ont mis en évidence qu'école et enseignants ne prennent en compte ni les langues d'origine des élèves ni leurs compétences plurilingues et pluriculturelles.

«Représentations de l'identité pluriculturelle et épanouissement scolaire. Au 'pays magique des plurilingues'», Giota GATSI, Olivier DELHAYE (p. 121-138).

L'école grecque, officiellement monolingue, ne tient compte ni de la compétence bilingue, ni de la langue d'origine des élèves immigrés. Des classes multiculturelles tentent de mettre en œuvre des pratiques éducatives renforçant l'identité plurilingue et pluriculturelle par le biais du développement de l'estime de soi des apprenants. Une analyse de contenu de la transcription d'entretiens conduits avec les élèves a permis de sélectionner de nouvelles pratiques de classe contribuant à l'épanouissement identitaire et au progrès scolaire sur la base des représentation que ces élèves ont de leur propre personne et de leurs apprentissages.

La Méthodologie, par les principes de l'éducation interculturelle orientée vers l'action, de la pédagogie différenciée, de l'interdépendance des langues au moment de la construction d'un répertoire ainsi que de la perspective actionnelle de l'usage et de l'apprentissage des langues. Les observations sont menées entre 2011 et 2013 dans une école primaire de la région de Thessalonique dans une classe du cours de renforcement de la connaissance de la langue grecque dont 75\% des élèves sont d'origine immigrée, classe qui se situe en dehors des heures normales d'enseignement: c'est «le pays magique ds plurilingues». Un double corpus est constitué: enregistrements d'entretiens spontanés avec ces élèves, leurs parents et leurs enseignants, de biographies langagières et de propos tenus dans un film crée à l'initiative des élèves. Deux 
Compte-rendu

A. TABOURET-Keller analyses, qualitative et quantitative, sont appliquées dont les résultats présentés distinguent:

Les représentations liées aux pratiques retenues

(1) Visite d'une mère d'élève migrante dans la classe

(2) Manifestations publiques auxquelles sont conviés les parents migrants

(3) Organisation de manifestations plurilingues et pluriculturelles dans l'école et hors de son enceinte

(4) Activité littéraire interculturelle

(5) Constitution de dictionnaires et de répertoires plurilingues

(6) Jeu théâtral en plusieurs langues

(7) Réaménagement de la classe

(8) Publication de recueils de textes identitaires

\section{Les représentations récurrentes dans les entretiens}

L'expression des représentations les plus significatives se retrouve dans les extraits extrêmement précieux d'entretiens conduits avec les élèves (les entretiens se sont déroulés en grec, la traduction en français est du fait des auteurs). Ces extraits concernent Ermal (10 ans), Sophie (12 ans), Koritsi-2 (12ans), Graciéla (10ans), Xénia (11 ans).

Représentations et biographies langagières: celles-ci sont faites par écrit et reproduits dans le texte, les extraits concernant Eduardos (11 ans), Kiki (11 ans), Siour (10 ans).

Discussion et conclusions: Les résultats de la recherche ont mis en évidence de nouvelles pratiques qui contribuent plus efficacement que d'autres à l'épanouissement identitaire et au progrès scolaire. Parmi les facteurs mis en évidence, sont mentionnés:

- une évolution des représentations initialement négatives vers des représentations plus positives concernant l'image des parents et des enfants migrants, l'intérêt porté par les parents migrants à l'éducation de leurs enfants, l'évacuation du stéréotype de l'enfant migrant à problèmes, la remise en question de l'idée que l'usage l'apprentissage simultané de plusieurs langues puisse perturber la scolarité d'un élève

- la substitution de l'identité plurilingue à l’identité migrante successivement déculpabilisés, admise, reconnue publiquement, puis affirmée

- la conscience des bienfaits de la déscolarisation de l'environnement d'apprentissage devenu alternatif

- la découverte par les élèves de nouvelles stratégies d'apprentissage renforcé par la multiplicité des gratifications, des références à la famille, au pays et à la culture d'origine, des possibilités d'emploi de la langue d'origine

- la constitution d'une nouvelle image de la compétence plurilingue qui fait retrouver aux élèves une dignité suffisante pour exprimer le désir de faire apprendre leur langue

- l'émergence d'une culture communautaire nouvelle avec le constat de 
Compte-rendu

A. TABouret-Keller l'existence d'une littérature dans toutes les cultures.

Au cours d'entretiens réalisés en fin d'année, les enseignants indiquent que les élèves ont enrichi leur répertoire lexical, comprennent les consignes des activités, s'expriment ave une plus grande aisance en grec (phrases complètes, correction grammaticale, orthographe). Leur entourage note qu'ils arrivent à produire un discours suivi, sans craindre de devoir recourir à l'alternance de codes ou de demander le sens d'un mot, et surtout qu'ils sont plus sûrs d'eux et beaucoup plus épanouis.

«Quatre approches didactiques pour la formation linguistique des migrants nouveaux arrivants», Michel GOUT (p. 139-153).

Aujourd'hui, en Europe, l'obligation institutionnelle d'«intégration linguistique» pour les migrants, avec la signature d'un contrat d'accueil et le passage de tests qui décident de leur régularisation administrative, produit un effet de stress ressenti par l'apprenant en formation linguistique qui s'avère contre-productif sur le plan pédagogique: des «filtres affectifs» tels que la motivation et la reconnaissance seraient nécessaires pour faciliter un retour de confiance. Dans un perspective neurologique l'affect fait bien partie de l'acte cognitif: affect et cognition fonctionneraient de manière inséparable. De plus, la réussite de l'apprentissage des langues étrangères dépendrait moins des matériaux, des techniques et des analyses linguistiques que de ce qui se passe dans et entre les personnes pendant la classe. Quatre approches didactiques observées dans différents pays sont rapportées:

(1) Les «textes d'identité» renforcent chez le migrant un sentiment de confiance: il a une histoire, une culture propre auxquelles le formateur porte intérêt ce qui établit une relation et lève les obstacles à l'apprentissage. Une expérience portant sur la création de textes bilingues par les élèves eux-mêmes dans un établissement scolaire canadien où plus de 40 nationalités sont représentées, illustre la dimension identitaire de la langue et la nécessité de prendre l'élève en considération dans sa globalité avec ses connaissances antérieures et son capital culturel.

(2) L'appropriation linguistique par la pratique artistique et culturelle: dans cette approche, actuellement en oeuvre en Belgique (Fédération WallonieBruxelles), le terme «appropriation» s'applique à toute formation non-formelle, par opposition à l'enseignement normatif de la langue. Liidée force qui sous-tend cette action, est que les pratiques culturelles ont un double effet bénéfique pour l'apprentissage linguistique des nouveaux arrivants: elles aident à fixer la matière vue pendant le cours et déclenchent une appropriation de la langue/culture. Cette approche est immersive, elle table sur la mise en œuvre de projets dont le but est de créer du plaisir par des supports ludiques dans l'apprentissage: théâtre, récit de contes, chant.

(3) La méthode de conscientisation de Paulo Freire (1): l'approche participati- 
Compte-rendu

A. TABouret-Keller ve de Freire vise à apprendre la langue tout en développant son esprit critique, à exercer sa liberté de penser et à juger pas soi-même afin d'avoir une conscience éclairée des situations. L'exemple présenté en détail est celui de l'application du thème de l'intégration tout au long de cinq semaines dans deux établissements de la banlieue londonienne: c'est par le tâtonnement expérimental et l'expression libre que l'apprenant fait sa découverte de l'anglais, l'expression se libérant d'autant plus facilement que le thème abordé rejoint son vécu profond. L'apprentissage est doublement orienté vers un but utilitaire (la langue) et un but réflexif (la prise de conscience d'un problème), le thème de l'intégration étant central. Celle-ci concerne le changement progressif des habitudes des personnes qui ont émigré et le besoin de maintenir leurs valeurs tout en pratiquant la langue d'accueil.

(4) La pédagogie des projets ne conçoit plus l'apprentissage comme une accumulation de connaissances décontextualisées mais comme la réalisation d'un projet par le groupe-classe: l'apprenant est associé de manière contractuelle à l'élaboration de ses savoirs. La classe est considérée comme un groupe social, et, comme «dans la vie» la liberté de communication est totale et non-contrainte: paralangage, gestuelle, intonations, mimétique, utilisation d'une langue proche. L'apprentissage collaboratif ne se fixe pas pour premier objectif l'apprentissage de la langue mais la réussite d'un projet de groupe par l'utilisation de la langue source. Les acquisitions que l'apprenant fait au cours de ces dynamiques de projets débordent et dépassent les aspects cognitifs, l'apprenant pouvant développer même partiellement des compétences interculturelles, de réflexivité, ou des qualités comme la persévérance et la solidarité.

\section{Quelques conditions pour la réussite de projets}

La pédagogie de projets demande un engagement total de l'apprenant qui doit percevoir la tâche à accomplir d'un grand intérêt pour luimême ou pour ses proches: «a wholehearted purposeful activity». Les exemples qui illustrent de telles réussites proviennent de France, d'Angleterre (différents quartiers) avec des populations d'origine différente. L'apprentissage de la langue n'est qu'une étape dans le processus d'émancipation (empowerment) qui doit conduire l'individu vers l'action au service de la société et de la défense de ses droits.

Conclusion: les méthodes décrites partent du principe de l'apprentissage cognitif qui ne peut se dérouler de façon satisfaisante que si l'apprenant se trouve dans une condition optimale de réception, elles s'inspirent de la philosophie constructiviste qui affirme que c'est l'apprenant qui construit lui-même son savoir, elles promeuvent en outre l'aspect collectif de l'apprentissage dans un cadre collaboratif par opposition à la forme individualisée de l'enseignement classique dans un cadre compé- 
Compte-rendu

A. TABouret-Keller titif: l'apprenant découvre de nouvelles perspectives et apprend à renouveler son rapport au monde, à développer son autonomie, et à amorcer son émancipation.

«Eveil aux langues et place des langues minorisées dans des projets de recherche-action en contexte scolaire portugais», Filomena MARTINS, Ana Isabel ANDRADE, Ana Sofia PINHO, Ana Raquel SIMÕES (p. 155-175).

Dans les années 1970, le Portugal s'ouvre à la circulation de populations de différents horizons géographiques, linguistiques, sociaux et culturels; dans les décennies suivantes, avec l'ouverture des frontières et la libre circulation des biens et des personnes, cette mobilité migratoire va s'accentuer. Dans le cadre des travaux du Conseil de l'Europe sur la didactique du plurilinguisme (Conseil de l'Europe, Strasbourg, 2009), on assiste à une prise de conscience croissante de la diversité linguistique et culturelle, principalement conséquence de la mondialisation, des avancées technologiques, des migrations, de l'internet. Mais cette prise de conscience du plurilinguisme se reflète-t-elle dans les pratiques d'éducation langagière?

Le contexte de la diversité linguistique périphérique est brièvement présenté avec pour exemple la région d'Aveiro qui, en 2014, compte 11984 étrangers (principalement du Brésil, d'Ukraine, de Chine, de Roumanie et du Venezuela). La prise de conscience de la place à accorder à une éducation à la diversité linguistique commence à peine à émerger comme un objet de recherche et d'intervention didactique face à une idéologie linguistique porteuse d'inégalités linguistiques et sociales: se pose alors la question de l'élaboration et de la mise en œuvre de programmes qui s'opposent au monolinguisme et au monoculturalisme.

Pour une formation à la diversité linguistique et culturelle: les expériences analysées se développent dans le cursus de formation des enseignants destinés à exercer à l'école maternelle et primaire dans le cadre d'un master de formation, ici détaillé, visant à développer des compétences professionnelles diverses, notamment de recherche en éducation à travers la conception, l'expérimentation et l'évaluation des projets collaboratifs de recherche-action sur le terrain, dans un monde «glocalisé» (sic), où le contact des langues est complexe et multiple.

Les objectifs du programme de formation sont de trois ordres: 1. familiariser à la connaissance de l'éducation à la diversité linguistique et culturelle dès les premières années de la scolarité, 2 . développer la compétence professionnelle des futurs enseignants dans le domaine d'éducation, 3. faire réfléchir sur son propre parcours de formation en veillant à mobiliser des expériences et des compétences (connaissances, capacités, attitudes) autour du rôle des langues et des cultures dans les situations d'éducation et/ou de formation. 
Compte-rendu

A. TABouret-Keller
Projets d'éveil aux langues et aux cultures: deux projets sont présentés en détail. Le premier (novembre, décembre 2013), intitulé «L'île des lettres», a été développé à l'école maternelle avec un groupe de neuf enfants, à une exception près, tous âgés de cinq ans, pendant trois semaines à raison de six séances en milieu semi-rural où il n'y avait aucun enfant d'immigré. De telles «classes de littératie sont remplies d'imprimés: livres, étiquettes, panneaux signalétiques, graphiques, mots, claviers et plein d'autres étalages du langage foisonnent» (p. 163). Les exercices proposés aux enfants sont détaillés (exploration de la bibliothèque, comparaison d'écritures - portugais, arabe, japonais jeu de domino avec des mots de ces trois langues, etc.)

Le second projet a été développé dans une classe de vingt élèves de l'école primaire d'un centre scolaire situé en milieu semi-rural (novembre, décembre 2013) ne comprenant que des enfants portugais ayant peu de contacts avec des enfants immigrés, la plupart des enfants faisant leurs premières expériences de lecture et d'écriture. L'entrée dans l'écrit est considéré comme un moment-clé de la scolarisation de l'enfant et de la formation des professeurs d'école primaire. Le projet d'éveil présenté est celui de deux étudiantes ayant retenu deux langues minoritaires et minorisées au Portugal, le cap-verdien et le tétum (langue polynésienne parlée au Timor oriental, où elle est langue officielle avec le portugais), avec comme objectifs de connaitre les représentations des enfants sur différentes langues et leurs locuteurs, notamment les langues introduites au Portugal via la migration (au début et à la fin des séances d'éveil aux langues). Travaillant avec des livres plurilingues, les professeurs «développent un rapport à la littéracie chez leurs élèves à partir de livres pratiques et de compétences plurilittératiées» pour faire référence aux pratiques et aux compétences développées dans plusieurs littéracies ou formes de littéracies dans une ou plusieurs langues: entre autre, l'apprentissage de la grammaire (la flexion en genre et en nombre, et leur construction/marquage en différentes langues, liée aux représentations des enfants à ce propos), de la notion d'arbitraire dans les phénomènes linguistiques (ici, le genre et le nombre); «le terme plurilittératie sous-tend alors l'idée que les langues et formes littéraciées se développent en relation les unes aux autres, de même qu'en relation à leurs contextes d'émergence et qu'elles constituent un atout et une ressource d'apprentissage» (p. 167). Différentes activités en application avec ces directives sont présentées en détail et aboutissent à la réflexion suivante.

Pour une évaluation de la formation. Quinze étudiantes ont rempli un questionnaire composé essentiellement de questions ouvertes portant sur les langues que doit proposer l'école portugaise dans ses programmes, sur la notion de «langue minoritaire», sur le rôle du professeur vis-à-vis de 
Compte-rendu

A. TABOURET-Keller telles langues, sur leur choix de langues, sur les moments marquants de leur formation. Les catégories thématiques suivantes ont émergé des énoncés analysés:

- langues, diversité linguistique et cursus scolaire: «les réponses manquent d'originalité»: l'école portugaise devrait placer l'anglais en première place dès l'école primaire, les langues minoritaires sont caractérisées comme langue régionale à petit nombre de locuteurs, et les langues minorisées par leur manque de prestige sociale et par le fait d'être en danger, réprimées par des mesures d'aménagement politique;

- rôle du professeur dans la valorisation des langues: «l'éveil aux langues devient une opportunité d'éveiller la curiosité des enfants, tout en cherchant à attirer leur attention pour l'égalité des langues tout en leur expliquant pourquoi il y a des langues qui sont moins parlées et qui sont minorisées et pourquoi il faut les valoriser», l'éveil aux langues est considérée comme une «matière-pont» des disciplines permettant au professeur de faire un travail interdisciplinaire. Pour leurs projets de recherche-action (planification, pas moins de dix-sept langues sont choisies par les étudiantes, tant parce qu'elles sont très éloignées du portugais avec des alphabets/systèmes d'écriture très différents que parce qu'elles sont moins présentes dans le quotidien;

- projet de recherche-action: il représente pour les étudiantes un parcours d'apprentissage de la profession, de valorisation de l'engagement et du compromis éthique, social, politique dans la formation des enfants, les éduquant dès les premières années de scolarité à valoriser la différence et la diversité, parcours qui conjoint la recherche théorique à l'analyse des données;

- formation: «'élaboration individuelle d'un mémoire, qui comporte un travail de recherche en éducation, avec soutenance publique devant un jury, constitue pour toutes une épreuve», et la confrontation à beaucoup de difficultés surtout avec la rédaction du cadre théorique. Le fait de devoir lire beaucoup de textes et de commencer à écrire apparait comme une tâche compliquée impliquant beaucoup de concentration, de recherche, d'apprentissage pour que «nous soyons capables de réfléchir et d'écrire sur ce que nous avons lu». Le stage en salle de classe est souligné comme opportunité indispensable d'apprentissage des techniques d'enseignement (planification et exécution), d'articulation entre les disciplines scolaires et de valorisation des langues et des expériences langagières des enfants. L'importance de l'école maternelle et des premières années de scolarité est soulignée pour l'influence qu'elle exerce sur la façon dont les enfants envisagent la réalité sociale, linguistique et culturelle. «Les étudiantes se forgent des sortes de «maximes» tels que: être attentive aux intérêts des enfants, développer l'auto-connaissance de ses limites, développer la connaissance des enfants, prendre en compte l'importance de la famille dans la socialisation des enfants, pouvoir compter sur les parents et d'autres personnes allophones dans l'activité d'éveil aux langues, affronter les problèmes des enfants (le divorce, l'émigration des parents), 


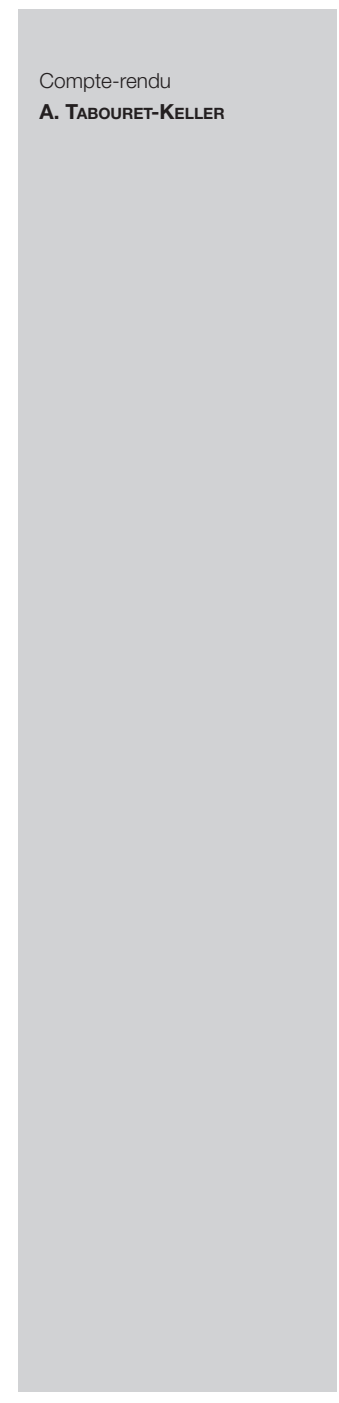

NOTE développer une conscience langagière plurilingue (pas seulement en portugais), risquer et oser aller plus loin avec les enfants» (p. 174).

Pour conclure: le compte rendu de ces expériences de terrain illustre un dispositif non pas exclusivement centré sur la langue de scolarisation et son apprentissage, mais sur une formation à une éducation langagière globale, pour tout public, y incluant les classes dites «monolingues». La formation dispensée prétend valoriser une conception d'enseignements intégrés, capable de situer chaque enseignement langagier ou d'autre nature par rapport aux autres matières, en mettant en relation des savoirs divers, dans la perspective d'un cursus scolaire où l'éveil aux langues et aux cultures pourrait avoir sa place, notamment à travers la problématique de la minorisation et de la stigmatisation linguistique. La recherche sur la formation des formateurs (enseignants mais aussi d'autres formateurs) a beaucoup avancé ces dernières années mais la conception et la mise en œuvre de pédagogies efficaces pour la formation des enseignants reste un défi pour les éducateurs et les chercheurs: «il s'agit notamment de promouvoir chez les enseignants, d'une part, le développement d'une vision plus globale et critique de la programmation scolaire capable de prendre en compte les objectifs sociaux de l'éducation et, d'autre part, la capacité de prendre les décisions pertinentes sur les programmes et de les mettre en œuvre tout en s'adaptant aux contextes et à leurs caractéristiques» (p. 175). Le travail de l'équipe des formateurs de l'Université d'Aveiro met en œuvre «une didactique plurielle ouverte à la diversité (intra- et inter-) de la langue de scolarisation, en ouvrant vers une pluralité de langues et de codes, ce qui permet d'organiser une formation généraliste et, simultanément, plus focalisée sur un thème particulier, ici l'éducation à la diversité linguistique et culturelle» (p. 174).

Paulo Freire (1921-1997) est un pédagogue brésilien, auteur d'une méthode d'alphabétisation qui repose sur la prise de conscience de sa condition sociale par celui qui apprend (note ajoutée par ATK). 